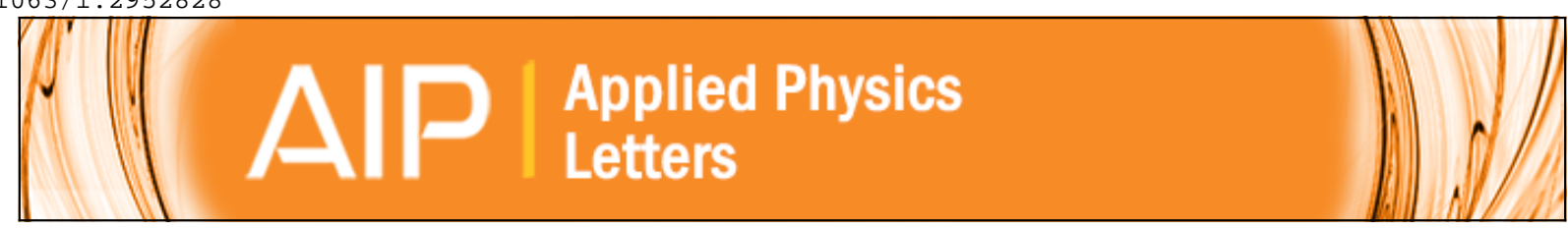

\title{
Giant microwave tunability in FeGaB/lead magnesium niobate-lead titanate multiferroic
} composites

J. Lou, D. Reed, C. Pettiford, M. Liu, P. Han, S. Dong, and N. X. Sun

Citation: Applied Physics Letters 92, 262502 (2008); doi: 10.1063/1.2952828

View online: http://dx.doi.org/10.1063/1.2952828

View Table of Contents: http://scitation.aip.org/content/aip/journal/apl/92/26?ver=pdfcov

Published by the AIP Publishing

\section{Over 700 papers \&} presentations on multiphysics simulation visw now

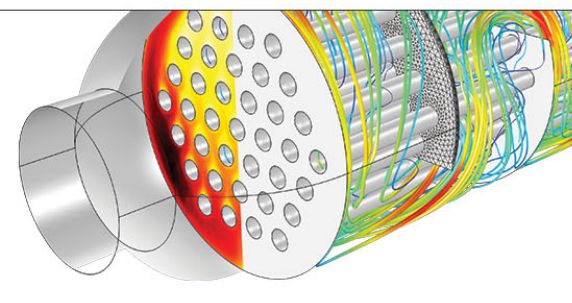




\title{
Giant microwave tunability in FeGaB/lead magnesium niobate-lead titanate multiferroic composites
}

\author{
J. Lou, ${ }^{1}$ D. Reed, ${ }^{1}$ C. Pettiford, ${ }^{1}$ M. Liu, ${ }^{1}$ P. Han, ${ }^{2}$ S. Dong, ${ }^{3, a)}$ and N. X. Sun ${ }^{1, b)}$ \\ ${ }^{1}$ Center for Microwave Magnetic Materials and Integrated Circuits (CM3IC), Department of Electrical \\ and Computer Engineering, Northeastern University, Boston, Massachusetts 02115, USA \\ ${ }^{2}$ H.C. Materials Corporation, Urbana, Illinois 61802, USA \\ ${ }^{3}$ Advanced Materials and Nanotechnology, College of Engineering, Peking University, Beijing 100871, \\ People's Republic of China
}

(Received 6 May 2008; accepted 9 June 2008; published online 30 June 2008)

\begin{abstract}
Giant magnetoelectric coupling at both microwave frequencies and dc was observed in an FeGaB/ lead magnesium niobate-lead titanate microwave multiferroic composite. A record high microwave frequency tunability of $\Delta f=900 \mathrm{MHz}$ or $\Delta f / f=58 \%$ was demonstrated with the change of an external electric field from -6 to $+2 \mathrm{kV} / \mathrm{cm}$. A strong electric field dependence of magnetic hysteresis loops was also observed. Such multiferroic composite provides great opportunities for electrostatically tunable microwave devices. (C) 2008 American Institute of Physics.
\end{abstract}

[DOI: 10.1063/1.2952828]

Multiferroic composite materials having two or more ferroic (ferroelectric, ferro/ferrimagnetic, antiferromagnetic, etc.) phases $^{1-3}$ have led to many devices such as picotesla sensitivity magnetoelectric (ME) magnetometers ${ }^{4,5}$ and electrostatically tunable microwave signal processing devices like resonators, ${ }^{6}$ phase shifters, ${ }^{7}$ filters, ${ }^{8}$ etc. Single crystal yttrium iron garnet (YIG) has been the magnetic material of choice for tunable multiferroic microwave devices ${ }^{6-8}$ due to its narrow ferromagnetic resonance (FMR) linewidth. However, the low saturation magnetostriction constant $\left(\lambda_{s}\right.$ $\sim 1.4 \mathrm{ppm}$ ) of YIG has severely limited the frequency tunability to be $\Delta f \leqslant 120 \mathrm{MHz}$ and $\Delta f / f \leqslant 2.5 \%$ and constrained the applications of these multiferroic based devices. While there are ferrites that have approximately two orders of magnitude higher $\lambda_{s}$ than YIG, e.g., $\mathrm{CoFe}_{2} \mathrm{O}_{4}$ has a $\lambda_{s}$ of 160 ppm, they show much wider FMR linewidth than that of YIG, leading to excessive microwave loss that is not suitable for microwave devices. In addition, ferrite materials have other disadvantages such as low saturation magnetization $\left(M_{s}\right)$, low permeability, the need for high processing temperatures of $\sim 1000{ }^{\circ} \mathrm{C}$, and large bias magnetic field for operation.

Multiferroic composite materials need to be optimized in all aspects, including individual magnetic phase, piezoelectric phase, mode of coupling, and the way magnetic field and electric field are applied in order to achieve strong ME coupling with large frequency tunability at microwave frequencies.

The magnetic phase in microwave multiferroic composites needs to have a low FMR linewidth, large $\lambda_{s}$, high permeability, and low saturation magnetic field. Metallic magnetic thin films grown by physical vapor deposition or sputtering can have excellent magnetic softness with low FMR linewidth when their thickness is less than the skin depth $\delta_{s}=\sqrt{2 \rho / \mu \omega}$, where $\rho$ is the resistivity, $\omega$ is the angular frequency, and $\mu$ is the permeability of the magnetic films. ${ }^{9-13}$ These metallic magnetic films show great potential

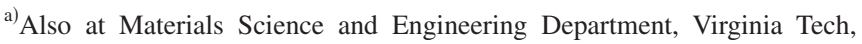
Blacksburg, VA 24061.

${ }^{b)}$ Electronic mail: nian@ece.neu.edu.
}

for low-cost integrated tunable microwave devices with low FMR linewidth, low processing temperature, high saturation magnetization, ${ }^{9,11-13}$ and much higher achievable $\lambda_{s}$, up to $1600 \mathrm{ppm}$ for Terfenol-D ${ }^{14}$ and $400 \mathrm{ppm}$ for FeGa (Galfenol) single crystals. ${ }^{15,16}$ However, metallic magnetic films bearing all of these properties are not readily available.

Most recently, we have reported a series of microwave magnetic thin film materials, the FeGaB films, ${ }^{9}$ with composition of $\left(\mathrm{Fe}_{100-y} \mathrm{Ga}_{y}\right)_{1-x} \mathrm{~B}_{x}(x=0-21$ and $y=9-17)$, which have large $\lambda_{s}$ and low saturation fields desired for multiferroic composite applications. The doping of boron into the giant magnetostrictive alloy FeGa changes the FeGaB films from polycrystalline to amorphous phase and leads to excellent magnetic softness with coercivity $<1$ Oe, narrow FMR linewidth of $16-20 \mathrm{Oe}$ at $X$-band $(9.6 \mathrm{GHz})$, large $\lambda_{s}$ of 50-70 ppm, high saturation magnetization of $11-15 \mathrm{kG}$, and a self-biased FMR frequency of $1.8 \mathrm{GHz}$. The combination of these properties makes the FeGaB films potential candidates for tunable ME microwave devices and other rf/ microwave magnetic device applications.

Lead magnesium niobate-lead titanate (PMN-PT) single crystal with $\langle 011\rangle$ poling was used as the ferroelectric phase in the microwave multiferroic composite in this work. Compare to the widely used lead zirconate titanate ceramics which have a typical piezoelectric coefficient $d_{31}$ of $\sim-200 \mathrm{pC} / \mathrm{N}$, PMN-PT single crystals with 28-32\% PT have a much larger $d_{31}$ of $\sim-1000 \mathrm{pC} / \mathrm{N}$ for $\langle 001\rangle$ poling and $\sim-1800 \mathrm{pC} / \mathrm{N}$ for $\langle 011\rangle$ poling. ${ }^{17}$ Another unique feature of the $\langle 011\rangle$ poled single crystal PMN-PT is its anisotropic in-plane piezoelectric coefficients of $d_{31}$ and $d_{32}$, with a negative $d_{31}$ of $\sim-1800 \mathrm{pC} / \mathrm{N}$ and a positive $d_{32}$ of $\sim 900 \mathrm{pC} / \mathrm{N} .^{17}$ The anisotropic in-plane piezoelectric coefficients of PMN-PT provide an exceptional opportunity for achieving large change in in-plane uniaxial anisotropy of the FeGaB films and therefore large FMR frequency tunability via inverse magnetoelastic coupling.

The multiferroic composites are designed to be a layered structure of epoxy-bonded $\mathrm{FeGaB} / \mathrm{Si}$ and PMN-PT single crystal using the L-T (longitudinal magnetized/transverse polarized) ME coupling mode. This L-T ME coupling mode takes full advantage of the large in-plane permeability of the 


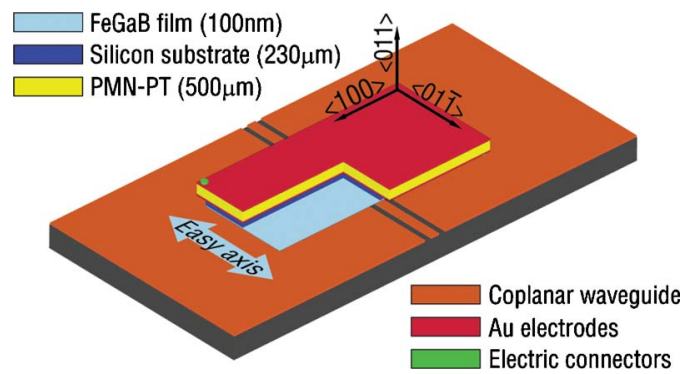

FIG. 1. (Color online) Schematic of sample configuration and microwave measurement setup.

magnetic films, and the anisotropic in-plane piezoelectric constants of PMN-PT, and exhibits a tunable FMR frequency range that is $\sqrt{\mu_{i}}$ times of that of the T-T mode. Assuming that the magnetic layer is directly bonded to the piezoelectric layer, and the thickness of the magnetic layer is much less than that of the piezoelectric layer, i.e., the substrate clamping effect from the Si substrate and the epoxy glue is neglected, we can readily get the effective anisotropic field induced by the ME coupling, following the inverse magnetoelastic relation, by

$$
\Delta H_{\mathrm{eff}}=3 \lambda_{s} Y d_{31} E / M_{s},
$$

in which $\Delta H_{\text {eff }}$ is the effective magnetic field induced by ME coupling and $Y$ is Young's modulus of the magnetic film. The induced FMR frequency change can be readily derived on the basis of the Kittel equation ${ }^{18}$ (cgs units)

$$
\Delta f_{\mathrm{FMR}}=\gamma \sqrt{\mu_{i}} \Delta H_{\mathrm{eff}},
$$

where $\gamma$ is the gyromagnetic constant of about $2.8 \mathrm{MHz} / \mathrm{Oe}$.

The amorphous FeGaB film with a thickness of $100 \mathrm{~nm}$ was used in our experiment, which was deposited on a $230 \mu \mathrm{m}$ thick (001) Si substrate at room temperature with a composition of $\mathrm{Fe}_{75} \mathrm{Ga}_{10} \mathrm{~B}_{15}$, saturation magnetization of $12.5 \mathrm{kG}$, initial permeability $\mu_{i}$ of $550-600$, and a $\lambda_{s}$ of $60 \mathrm{ppm}$. The FeGaB was then epoxy bonded to a $0.5 \mathrm{~mm}$ thick $\langle 011\rangle$ poled PMN-31\%PT single crystal that has a dimension of $5 \mathrm{~mm}$ (along its $\langle 01 \overline{1}\rangle$ direction) by $10 \mathrm{~mm}$ (along its $\langle 100\rangle$ direction). Also, the magnetic easy axis of the FeGaB film was placed parallel to the $\langle 01 \overline{1}\rangle$ direction of the PMN-PT to minimize the magnetic loss tangent, as shown in Fig. 1.

The ME coupling of the FeGaB/PMN-PT multiferroic composite at microwave frequencies was characterized on a customer made permeameter which consisted of a coplanar waveguide connected to the two ports of a vector network analyzer. The permeability spectra of the FeGaB/PMN-PT multiferroic composite under different electric fields across the thickness of the PMN-PT and under an external dc magnetic bias field of $\sim 20$ Oe applied parallel to the magnetic easy axis of the FeGaB film were extracted following the procedure described in Ref. 19, as shown in Fig. 2. We can see that the peak FMR frequency changes dramatically with external electric field, being $1.1 \mathrm{GHz}$ at $-6 \mathrm{kV} / \mathrm{cm}, 1.6 \mathrm{GHz}$ at zero field, and $2.0 \mathrm{GHz}$ at the coercive field of the PMN-PT single crystal, $+2 \mathrm{kV} / \mathrm{cm}$. This equals a tunable frequency $\Delta f=900 \mathrm{MHz}$ and $\Delta f / f_{0}=58 \%$, which is almost one order of magnitude higher than reported results and can be interpreted by the Kittel Equation ${ }^{18}$ (cgs units)

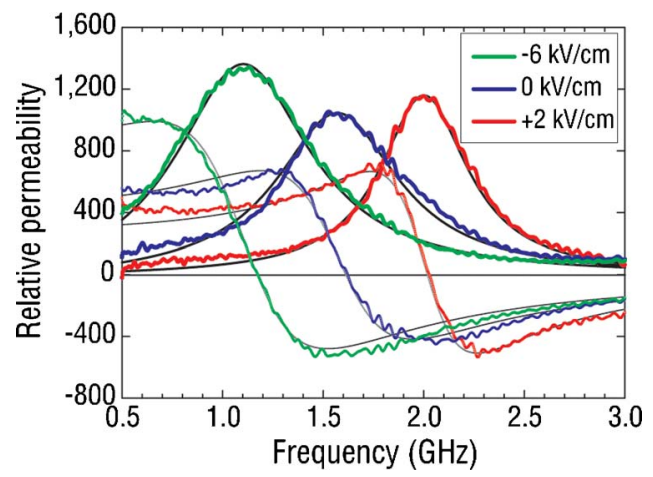

FIG. 2. (Color online) Permeability spectra under different electric fields. Black lines denote simulated results according to the Landau-LifshitzGilbert equation.

$$
f_{\mathrm{FMR}}=\gamma \sqrt{\left(H_{k}+H_{\mathrm{dc}}+\Delta H_{\mathrm{eff}}\right)\left(4 \pi M_{s}+H_{k}+H_{\mathrm{dc}}+\Delta H_{\mathrm{eff}}\right)},
$$

where $H_{\mathrm{dc}}$ is the external bias field and $H_{k}$ is the intrinsic in-plane anisotropy field of the FeGaB film.

It is notable that the full width at half maximum of the imaginary permeability spectrum is minimized at $0.5 \mathrm{GHz}$ at the PMN-PT coercive field of $2 \mathrm{kV} / \mathrm{cm}$ and maximized at $0.8 \mathrm{GHz}$ at $-6 \mathrm{kV} / \mathrm{cm}$ from which the FMR linewidth is calculated to be between 19 and 21 Oe. Besides the FMR frequency change, the initial relative permeability was also changed accordingly, from $\sim 1000$ for $-6 \mathrm{kV} / \mathrm{cm}$ to $\sim 350$ for $+2 \mathrm{kV} / \mathrm{cm}$, which can be easily understood by the following relation (cgs units):

$$
\mu_{i}=\frac{4 \pi M_{s}}{H_{k}+H_{\mathrm{dc}}+\Delta H_{\mathrm{eff}}} .
$$

The Landau-Lifshitz-Gilbert equation that governs magnetodynamic ${ }^{20}$ was used to fit these permeability spectra shown in Fig. 2 to extract $H_{k}+\Delta H_{\text {eff }}$, the total effective magnetic anisotropy fields excluding the applied bias field of $20 \mathrm{Oe}$, and the Gilbert damping constant $\alpha$. The total effective magnetic anisotropy fields from the ME coupling show a strong electric field dependence and are in the range of -6-21.5 Oe, which are shown in Fig. 3. The Gilbert damping constant shows a similar strong dependence of the electric field, which is 0.0235 at $-6 \mathrm{kV} / \mathrm{cm}$ and 0.015 with a $+2 \mathrm{kV} / \mathrm{cm}$ electric field. There is a clear trend that the Gilbert damping constant $\alpha$ drops with the increase in the FMR frequency or an increase in the effective magnetic anisotropy field from the ME coupling.

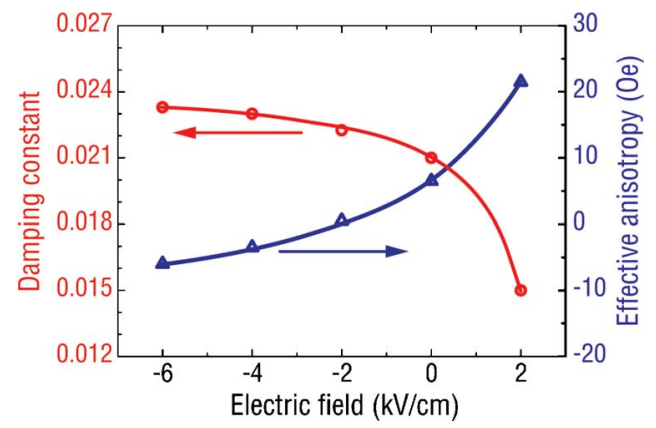

FIG. 3. (Color online) Calculated damping constant and total effective inplane anisotropy field (excluding the applied bias field of $20 \mathrm{Oe}$ ). 


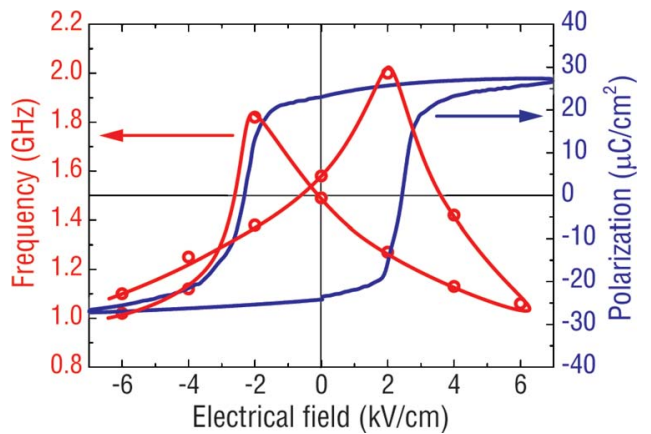

FIG. 4. (Color online) The "butterfly" shaped hysteresis of the FMR frequency vs electric field, and the ferroelectric $P$ - $E$ hysteresis loop.

The FMR frequencies are shown in Fig. 4 as a function of the applied electric fields; a clear hysteresis loop can be observed with a "butterfly" shape. This butterfly shape of the FMR frequency resembles the widely observed piezoelectric strain versus electric field butterfly curves for piezoelectric materials ${ }^{21}$ and matches the ferroelectric $P-E$ hysteresis loop of PMN-PT single crystal as well, which is also shown in Fig. 4. This once again confirms that the change of FMR frequency of the FeGaB film results from the ME coupling induced strain in the FeGaB film as described in Eq. (1).

Electric field induced change in magnetic hysteresis loops, which show the dc magnetic properties of the $\mathrm{FeGaB}$ film, was measured by vibration sample magnetometer and is shown in Fig. 5. The remnant magnetization ratio shows a remarkable change from $16 \%$ to $74 \%$ when the electric field was varied from -6 to $+2 \mathrm{kV} / \mathrm{cm}$. The clear trend of aniso-

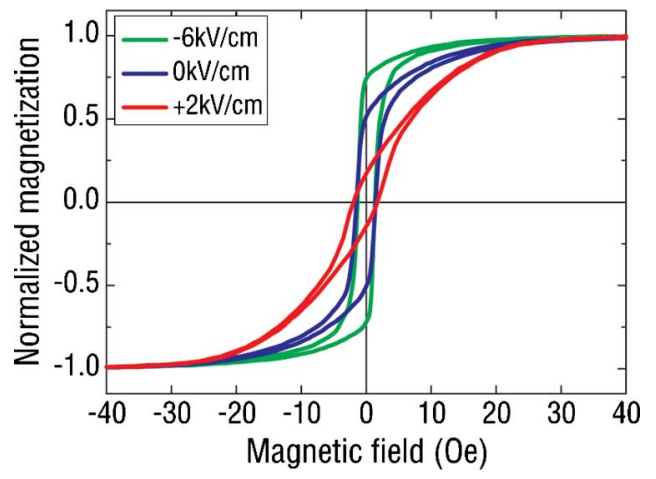

FIG. 5. (Color online) Magnetic hysteresis loops under different electric fields. tropy field change, that $-6 \mathrm{kV} / \mathrm{cm}$ electric field corresponds to a minimal anisotropy field while electric field of $+2 \mathrm{kV} / \mathrm{cm}$ provides a much higher anisotropy field, agrees well with the results from microwave measurements.

In conclusion, we have demonstrated a giant ME coupling at microwave frequencies and at dc in a $\mathrm{FeGaB} /$ PMN-PT ME composite, which shows a record high microwave frequency tunability of $\Delta f=900 \mathrm{MHz}$ or $\Delta f / f=58 \%$ with the change of external electric field from -6 to $+2 \mathrm{kV} / \mathrm{cm}$. Such multiferroic composite provides great opportunities for electrostatically tunable microwave devices.

This work was sponsored by NSF under Award Nos. ECCS-0746810 and DMR-0603115, ONR Award Nos. N000140710761 and N000140810526, and by Draper Laboratory through University UR\&D program.

${ }^{1}$ M. Fiebig, J. Phys. D: Appl. Phys. 38, R123 (2005).

${ }^{2}$ W. Eerenstein, N. D. Mathur, and J. F. Scott, Nature (London) 442, 759 (2006).

${ }^{3}$ C. W. Nan, M. I. Bichurin, S. X. Dong, D. Viehland, and G. Srinivasan, J. Appl. Phys. 103, 031101 (2008).

${ }^{4}$ S. X. Dong, J. F. Li, and D. Viehland, Appl. Phys. Lett. 83, 2265 (2003).

${ }^{5}$ J. Zhai, Z. Xing, S. X. Dong, J. F. Li, and D. Viehland, Appl. Phys. Lett. 88, 062510 (2006).

${ }^{6}$ Y. K. Fetisov and G. Srinivasana, Appl. Phys. Lett. 88, 143503 (2006).

${ }^{7}$ A. Ustinov, G. Srinivasan, and B. A. Kalinikos, Appl. Phys. Lett. 90, 031913 (2007).

${ }^{8}$ C. Pettiford, S. Dasgupta, J. Lou, S. D. Yoon, and N. X. Sun, IEEE Trans. Magn. 43, 3343 (2007).

${ }^{9}$ J. Lou, R. E. Insignares, Z. Cai, K. S. Ziemer, M. Liu, and N. X. Sun, Appl. Phys. Lett. 91, 18254 (2007).

${ }^{10}$ C. E. Patton, J. Appl. Phys. 39, 3060 (1968).

${ }^{11}$ C. Scheck, L. Cheng, I. Barsukov, Z. Frait, and W. E. Bailey, Phys. Rev. Lett. 98, 117601 (2007).

${ }^{12}$ S. X. Wang, N. X. Sun, M. Yamaguchi, and S. Yabukami, Nature (London) 407, 150 (2000).

${ }^{13}$ N. X. Sun, S. X. Wang, T. J. Silva, and A. B. Kos, IEEE Trans. Magn. 38, 146 (2002).

${ }^{14}$ G. Engdahl and I. D. Mayergoyz, Handbook of Giant Magnetostrictive Materials (Academic, New York, 2000), pp. 209-217.

${ }^{15}$ A. E. Clark, J. B. Restorff, M. Wun-Fogle, T. A. Lograsso, and D. L. Schlagel, IEEE Trans. Magn. 36, 3238 (2000).

${ }^{16}$ N. Srisukhumbowornchai and S. Guruswamy, J. Appl. Phys. 92, 5371 (2002)

${ }^{17}$ P. Han, W. Yan, J. Tian, X. Huang, and H. Pan, Appl. Phys. Lett. 86, 052902 (2005).

${ }^{18}$ C. Kittel, Phys. Rev. 73, 155 (1948).

${ }^{19}$ Y. Ding, T. J. Klemmer, and T. M. Crawford, J. Appl. Phys. 96, 2969 (2004).

${ }^{20}$ T. L. Gilbert, Phys. Rev. B 100, 1243 (1955).

${ }^{21}$ S. Park and T. R. Shrout, J. Appl. Phys. 82, 1804 (1997). 